\title{
The role of acylated-ghrelin in the regulation of sucrose intake
}

\author{
Tetsuya Kouno, Nobuteru Akiyama, Takahito Ito, Kumiko Fujieda, Isamu Nanchi, Tomohiko Okuda, \\ Mitsuru Notoya, Takanori Iwasaki and Hideo Yukioka
}

Shionogi Pharmaceutical Research Center, Shionogi \& Co., Ltd., Osaka, Japan

\begin{abstract}
The octanoyl modification of ghrelin by ghrelin $O$-acyltransferase (GOAT) is essential for exerting its physiologic actions. Since exogenous acylated-ghrelin has shown to stimulate food intake in humans and rodents, GOAT has been regarded as a promising target for modulating appetite, thereby treating obesity and diabetes. However, GOAT-knockout (KO) mice have been reported to show no meaningful body weight reduction, when fed a high-fat diet. In this study, we sought to determine whether GOAT has a role in the regulation of body weight and food intake when fed a dietary sucrose. We found that GOAT KO mice showed significantly reduced food intake and marked resistance to obesity, when fed a high-fat + high-sucrose diet. In addition, GOAT KO mice fed a medium-chain triglyceride (MCT) + high-sucrose diet showed a marked resistance to obesity and reduced feed efficiency. These results suggest that blockade of acylated-ghrelin production offers therapeutic potential for obesity caused by overconsumption of palatable food.
\end{abstract}

Key words: Ghrelin $O$-acyltransferase, Acylated-ghrelin, High-sucrose diet

GHRELIN, a 28-amino-acid peptide hormone, is produced in stomach, and modified by ghrelin $O$-acyltransferase (GOAT) to become acylated-ghrelin (AG). A number of studies have so far demonstrated that exogenous injection of AG, but not unacylated-ghrelin, stimulates appetite in humans and rodents [1-4], suggesting that ghrelin/GOAT system may function as an appetite controlling endocrine system. However, the role of endogenous AG in the regulation of food intake and body weight still remains elusive. For instance, ghrelin- or GOAT-knockout (KO) mice, when fed a high-fat diet or standard chow, have shown no significant change in food intake or body weight compared to WT mice [5-8]. On the other hand, when fed a medium-chain triglyceride (MCT) diet rich in a substrate for acylation of ghrelin, GOAT $\mathrm{KO}$ mice exhibited slightly but significantly reduced body weight compared to WT mice [9]. Moreover, recent studies have indicated that when fed a diet rich in sucrose or carbohydrate, GOAT KO mice showed less energy intake and body weight gain than WT mice $[10,11]$. Thus, endogenous ghrelin/GOAT system has been suggested to regulate systemic energy homeosta-

Correspondence to: Tetsuya Kouno, Shionogi Pharmaceutical Research Center, Shionogi \& Co., Ltd., 3-1-1 Futaba-cho, Toyonaka, Osaka, 561-0825, Japan.

E-mail: tetsuya.kouno@shionogi.co.jp

(C)The Japan Endocrine Society sis in specific dietary conditions. To access the role of endogenous ghrelin in the regulation of systemic energy homeostasis under several dietary conditions with a high-fat diet, a high-fat + high-sucrose diet, an MCT diet, and an MCT + high-sucrose diet, the present study was carried out to compare the difference in food intake, body weight, and feed efficiency between WT mice and GOAT KO mice. Our findings indicate that the physiological level of AG may be involved in the pathogenesis of obesity by regulating not only energy intake but also energy storage.

\section{Materials and Methods}

\section{Animals and housing}

GOAT-deficient mice were generated by Shionogi \& Co., Ltd as described previously [10]. The mice were given free access to water and housed in individual cages in a temperature-controlled environment at 20 to $23^{\circ} \mathrm{C}$ on a 12 -hour light/dark cycle. All experiments were conducted in a facility at Shionogi Pharmaceutical Research Center (Osaka, Japan) accredited by the Association for Assessment and Accreditation of Laboratory Animal Care International (AAALAC). All experimental protocols were approved by the Shionogi Pharmaceutical Research Center Institutional Animal Care and Use Committee. 


\section{Body weight and food intake}

Seven-week-old WT and GOAT KO mice were given free access to water and one of the diets, a high-fat diet (D12492), a high-fat + high-sucrose diet (D12445), an MCT diet (D12331), and an MCT + high-sucrose diet (D12327), and body weight and food intake were monitored once a week for 12 weeks. Feed efficiency was determined by body weight gain for 12 weeks (g) divided by cumulative energy intake for 12 weeks (kcal).

\section{Results and Discussion}

Consistent with the previous report $[7,8]$, when fed a high-fat diet, GOAT KO mice showed comparable body weight gain, food intake, and feed efficiency to WT mice (Fig. 1). On the other hand, when fed a high-fat + high-sucrose diet, GOAT KO mice showed a significant reduction in food intake and body weight with no change in feed efficiency compared to WT mice. These results suggest that ghrelin/GOAT system is involved in the overconsumption of sugar-sweetened foods associated with body weight gain in WT mice. In addition, when fed an MCT diet, GOAT KO mice showed a slight reduction in food intake and body weight with no change in feed efficiency compared to WT mice. Since the ingestion of MCT diet has been indicated to increase the blood levels of AG [12], it might be possible to consider that activation of ghrelin/GOAT system by feeding an MCT diet may contribute to food intake and weight gain in WT mice. Interestingly, when fed an $\mathrm{MCT}+$ high-sucrose diet, GOAT KO mice showed a remarkable reduction of food intake and body weight as well as reduced feed efficiency compared to WT mice, which can be explained by the previous finding that GOAT KO mice fed an MCT + high-sucrose diet had a significant increase in whole-body energy expenditure along with decreased energy intake [10]. Further experiments are needed to investigate the difference of physiological role of $A G$ in systemic energy homeostasis between an MCT + high-sucrose diet and other diets.

\section{Conclusions}

Our findings demonstrate that endogenous AG generated by GOAT system can promote the consumption of high-calorie food, especially containing high
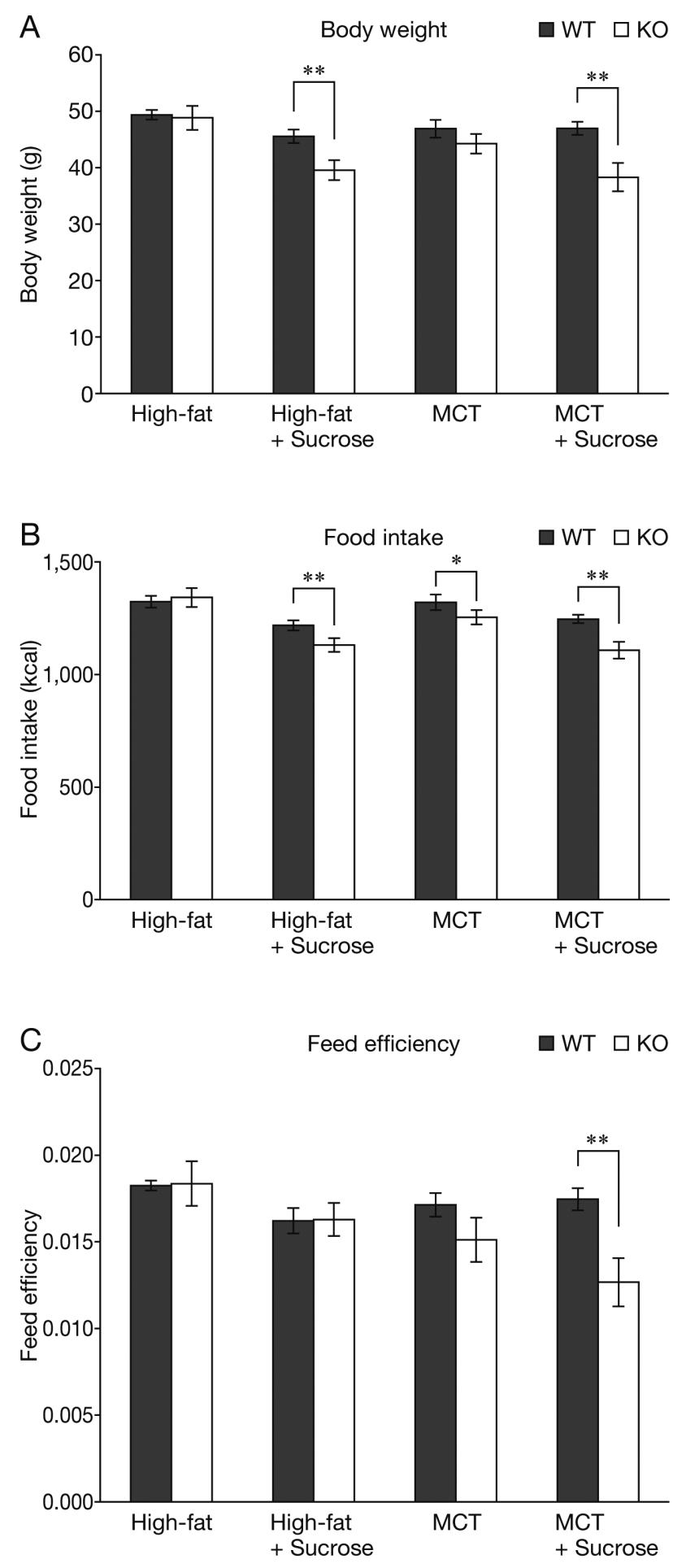

Fig. 1 A, Body weight of WT and GOAT KO mice after 12 weeks on one of the diets. B, Total calorie intake for 12 weeks. C, Feed efficiency determined by body weight gain divided by food intake. Data are presented as mean \pm SEM. ${ }^{*} p<0.05, * * p<0.01$ denotes the difference between WT and GOAT KO mice. 
amounts of sucrose, leading to a body weight gain. Recently, it has been reported that increasing consumption of sugar-sweetened beverages are associated with overweight and obesity [13], and a newly updated guideline from the World Health Organization (WHO) strongly recommends reducing the intake of free sugars such as sucrose and fructose [14]. Taking into consideration social demands to cut down on sugar intake, inhibiting GOAT activity may provide a therapeutic benefit in patients with obesity and type- 2 diabetes.

\section{References}

1. Neary NM, Small CJ, Wren AM, Lee JL, Druce MR, et al. (2004) Ghrelin increases energy intake in cancer patients with impaired appetite: acute, randomized, placebo-controlled trial. J Clin Endocrinol Metab 89: 2832-2836.

2. Wren AM, Seal LJ, Cohen MA, Brynes AE, Frost GS, et al. (2001) Ghrelin enhances appetite and increases food intake in humans. $J$ Clin Endocrinol Metab 86: 5992-5995.

3. Nakazato M, Murakami N, Date Y, Kojima M, Matsuo $\mathrm{H}$, et al. (2001) A role for ghrelin in the central regulation of feeding. Nature 409: 194-198.

4. Tschop M, Smiley DL, Heiman ML (2000) Ghrelin induces adiposity in rodents. Nature 407: 908-913.

5. Sato T, Kurokawa M, Nakashima Y, Ida T, Takahashi T, et al. (2008) Ghrelin deficiency does not influence feeding performance. Regul Pept 145: 7-11.

6. Sun Y, Ahmed S, Smith RG (2003) Deletion of ghrelin impairs neither growth nor appetite. Mol Cell Biol 23: 7973-7981.

7. Wortley KE, Anderson KD, Garcia K, Murray JD, Malinova L, et al. (2004) Genetic deletion of ghrelin does not decrease food intake but influences metabolic fuel preference. Proc Natl Acad Sci U S A 101: 8227-8232.

8. Zhao TJ, Liang G, Li RL, Xie X, Sleeman MW, et al.
(2010) Ghrelin $O$-acyltransferase (GOAT) is essential for growth hormone-mediated survival of calorie-restricted mice. Proc Natl Acad Sci U S A 107: 7467-7472.

9. Kirchner H, Gutierrez JA, Solenberg PJ, Pfluger PT, Czyzyk TA, et al. (2009) GOAT links dietary lipids with the endocrine control of energy balance. Nat Med 15: 741-745.

10. Kouno T, Akiyama N, Ito T, Okuda T, Nanchi I, et al. (2016) Ghrelin $O$-acyltransferase knockout mice show resistance to obesity when fed high-sucrose diet. $J$ Endocrinol 228: 115-125.

11. Kouno T, Akiyama N, Fujieda K, Nanchi I, Okuda T, et al. (2016) Reduced intake of carbohydrate prevents the development of obesity and impaired glucose metabolism in ghrelin $O$-acyltransferase knockout mice. Peptides 86: 145-152.

12. Nishi Y, Hiejima H, Hosoda H, Kaiya H, Mori $\mathrm{K}$, et al. (2005) Ingested medium-chain fatty acids are directly utilized for the acyl modification of ghrelin. Endocrinology 146: 2255-2264.

13. Bermudez OI, Gao X (2010) Greater consumption of sweetened beverages and added sugars is associated with obesity among US young adults. Ann Nutr Metab 57: 211-218.

14. World Health Organization. Sugars intake for adult and children. Guideline 2015. 